of the river's development. Palaeobotanical evidence (from macroremains, such as fruits and seeds, and from pollen) is also helpful and has been analysed by $\mathrm{K}$. Tobolski (University of Poznan).

The outer, larger series of palaeomeanders contains botanical evidence of an origin during the closing phases of the last glaciation. The pollen spectra are herb dominated and the major tree components are birch and pine. The dwarf birch (Betula nana), juniper, Artemisia and Chenopodiaceae all provide evidence suggestive of open, park-tundra vegetation typifying late-glacial conditions. Precise location of the sediments within the lateglacial chronology is difficult, on the palaeobotanical evidence, and one wonders whether the argument about whether the basal deposits date from before or after the $B \phi$ lling Interstadial can ever be resolved on this basis. The observed changes in sediment and pollen stratigraphy could so easily be a consequence of inwash from the steep sides of the channels, thus confusing the stratification of the deposits.

Radiocarbon dating has been attempted and figures in excess of $11,000 \mathrm{BP}$ have been obtained for certain levels, but the sediments are calcareous, making the precise interpretation of such dates difficult. It is reasonable, however, to conclude that the development of the wider series of meanders (which replaced the braided system) occurred during the lateglacial. This could be accounted for by the reduced flow resulting from the completion of ice wastage, the development of a complete (and partly wooded) vegetation cover, and the possibility of reduced precipitation.

The inner series of meanders proved to be considerably younger. The pollen spectra of the basal sediments within these are tree dominated, with oak predominant. Available radio-carbon dates suggest that some of these younger palaeomeanders began forming around 4,000 years ago, and some later than this. The smaller radii suggest that more water was moving down the Warta, but whether this was a consequence of climatic changes or human deforestation is impossible to ascertain.

The enigma of man's role in the palaeohydrology of central Poland is perhaps epitomized by the remarkable excavations of an Iron Age fortified settlement at Biskupin near Bydgoszcz. Built upon a low-lying peninsula extending into a 100 hectare lake this timber-built settlement, dating from $550 \mathrm{BC}$, has been extraordinarily well preserved as a consequence of raised lake levels swamping the wooden foundations. 35,000 stakes of oak and pine formed a palisade around the camp, which covered an area of about 2 hectares and within which were rows of wooden buildings, over 100 in all. The population was largely agricultural, growing wheat, barley, millet, beans, lentils and flax, and this arable emphasis, coupled with the timber necessary to build such a construction, must have involved extensive deforestation of the surrounding region. Which brings one back to the question of whether such forest clearance could have affected the hydrological regime in such a way as to lead to raised lake levels and the ultimate swamping of the settlement which finally led to its abandonment. Since this particular period in prehistory is generally believed to have been a time of increasing precipitation and lowered temperatures in northern Europe, this may have to remain just another palaeohydrological conundrum.

\section{A particularly anomalous Seyfert galaxy}

\section{from J.H. Krolik}

SEyfert galaxies and their quasar cousins have provided much observational mash for the vats of theoretical speculation over the years since their discovery. A recent article by $\mathrm{T}$. Heckman and B. Balick' has thrown an especially juicy bucketful into this already potent brew. Ordinarily, what astronomers find extraordinary in these objects are the very large continuum luminosity $\left(10^{43}-10^{45} \mathrm{erg} \mathrm{s}^{-1}\right.$ in Seyferts, $10^{45}-10^{47} \mathrm{erg} \mathrm{s}^{-1}$ in quasars) and the very strong, broad $\left(10^{3}-10^{4} \mathrm{~km} \mathrm{~s}^{-1}\right)$ emission lines coming from an optically unresolved source believed to be no more than a few parsecs in size. In this new paper, attention is turned to extraordinary goings-on outside the nucleus of a Seyfert galaxy.

Heckman and Balick made vidicon pictures of the galaxy Markarian 335 with a series of interference filters covering bands centred on $\mathrm{H} \alpha$ and $[\mathrm{O}$ III] $\lambda 5007$, each $3,300 \mathrm{~km} \mathrm{~s}^{-1}$ wide (FWHM), and extending in total over a range $\pm 5,000 \mathrm{~km} \mathrm{~s}^{-1}$ from line centre. Most of the line emission does indeed come from the galactic nucleus, but surprisingly, there is a significant flux in most of the observed wavelength range from regions clearly separate from the nucleus. Interpreting the emission as coming from a large photoionized nebula, the inferred mass of gas outside the nucleus is $\sim 2 \times 10^{\times} n_{\mathrm{c}}^{-1} M_{\mathrm{c}}$ and its

J.H. Krolik is in the Harvard Center for Astrophysics, Cambridge, Massachusetts. kinetic energy $\sim 2 \times 10^{58} n_{\mathrm{e}}^{-1} \mathrm{erg}$, where $n_{\mathrm{e}}$ is its electron density in $\mathrm{cm}^{-3}$. Such a large mass of gas moving at such high speed has never been seen elsewhere.

The first question that comes to mind is the source of ionization to drive the $\mathrm{H} \alpha$ recombination radiation. Heckman and Balick check that it is plausible for the Seyfert nucleus to be supplying enough ionizing photons. Baldwin and colleagues $^{2}$ previously showed that the nucleus of $3 \mathrm{C} 120$ superionizes its $\mathrm{H}$ II regions. Here the Seyfert nucleus has a much more dramatic influence on its galactic environment.

The second question is the source of the gas. An obvious supposition, and one that Heckman and Balick consider, is that this gas is material that once radiated the nuclear broad emission lines but has now spread further out into the galaxy. Unfortunately, we have no clear way of telling whether the nuclear emission line clouds travel radially in, radially out, or go in any other direction. Some lines (for example, C IV $\lambda 1549$ ) have profiles which are easy to measure, but are emitted isotropically, and so tell us nothing about cloud kinematics. On the other hand, those lines, such as $\mathrm{L} \alpha$, which are emitted only from one face of a cloud ${ }^{3}$ are often so distorted by blending or intervening absorption that their usefulness is destroyed. Therefore, the presence of this gas may be as good an indication that at least some Seyfert nuclei expel gas some of the time as asymmetry of nuclear emission line profiles.
Once the possibility of a nuclear origin for this gas is admitted, many other questions instantly arise. Why did the expulsion last only for $10^{6}$ years (its kinematic age)? Seyfert galaxies are generally supposed to live at least $10^{8}$ years. Why did it occur only in Markarian 335, a galaxy which doesn't appear remarkable in any other way (but one, which we discuss in the following paragraph). In their kiloparsecs-long passage out into the galaxy, why haven't the clouds been appreciably slowed by drag? Why is the shape of the nebula so strongly asymmetric?

The final, and perhaps most interesting, question stems from Heckman and Balick's measurement of the radial profile of the galaxy's continuum emission. They find that the surface brightness declines away from the centre in a way more characteristic of elliptical galaxies than spirals. If Markarian 335 is an elliptical galaxy, it would be the only such Seyfert galaxy known; all other Seyfert galaxies whose types have been determined are spirals. Ordinarily, when an elliptical galaxy has a line-emitting nucleus, it is also a bright radio source, which Markarian 335 is certainly not. On the other hand, Seyfert galaxies sometimes do evince small-scale radio emission near their cores $^{4}$. VLA observations of this unusual Seyfert galaxy might be a particulary fruitful next set.

Heckman \& Balick, B. Astrophys. J. 247. 32 (1981) Baldwin et at. Astrophys J. 236, $388(1980)$. Kwan \& Krolik Astrophys. J. (in the press). Wilson \& Willis Astrophys. J. 240, 429 (1980) 